\title{
Current status and progress of lymphoma management in China
}

\author{
Yuankai Shi ${ }^{1}$
}

Received: 5 December 2017 / Revised: 8 January 2018 / Accepted: 15 January 2018 / Published online: 31 January 2018

(c) The Japanese Society of Hematology 2018

\begin{abstract}
Lymphoma is a large group of lymphoid hematopoietic malignancies including Hodgkin lymphoma and non-Hodgkin's lymphoma. The various subtypes of lymphoma are different in clinical features, response to treatment and prognoses. The relative frequency of specific subtypes of lymphoma varies geographically. The mature $\mathrm{T}$ cell lymphoma is much more common in East Asia compared with Western countries. Chemotherapy plays an important role in the treatment of lymphoma. With advances in understanding the biology and genetics of lymphoma, many new agents are used in the treatment of lymphoma. In mainland China, some new agents and new combination chemotherapy regimens showed high efficacy and good tolerability. Chidamide, a histone deacetylase inhibitor, has been approved for the treatment of relapsed or refractory peripheral $\mathrm{T}$ cell lymphoma by the China Food and Drug Administration. Anti-programmed death 1 antibodies and chimeric antigen receptor-engineered $\mathrm{T}$ cells have been explored for lymphoma immunotherapy in Chinese patients. Advances in the treatment have substantially increased the likelihood of cure for patients with lymphoma.
\end{abstract}

Keywords Lymphoma $\cdot$ Chemotherapy $\cdot$ New agents

\section{Introduction}

Lymphoma is a diverse group of malignancies that originates from either $\mathrm{B}, \mathrm{T}$ or NK cells. According to the cancer statistics in 2013, the incidence of lymphoma was 4.2 per 100,000 and the mortality was 2.2 per 100,000 in mainland China [1], making it the eleventh most common cancer and the tenth leading cause of cancer death [2]. There are some differences in the distribution of lymphoma subtypes between East Asia and Western countries. The mature T/ NK cell neoplasms display higher rates on the Asian continent than others $[3,4]$. In mainland China, diffuse large B cell lymphoma (DLBCL) is still the most common subtype of lymphoma. Extranodal NK/T cell lymphoma, nasal type (ENKTL) is a common subtype and makes up $11-15 \%$ of all lymphoma [3, 4]. The treatment modalities for lymphoma

Yuankai Shi

syuankai@cicams.ac.cn

1 Department of Medical Oncology, Beijing Key Laboratory of Clinical Study on Anticancer Molecular Targeted Drugs, National Cancer Center/Cancer Hospital, Chinese Academy of Medical Sciences and Peking Union Medical College, No. 17 Panjiayuan Nanli, Chaoyang District, Beijing 100021, China include chemotherapy, radiotherapy, or their combinations. High-dose chemotherapy combined with autologous stem cell transplantation (HDC/ASCT) is still an important treatment strategy for high-risk or relapsed patients. Furthermore, advances in genetic analysis will provide new approaches in molecular targeted therapy. These new developments will improve the outcome of lymphoma patients.

\section{Hodgkin lymphoma}

Hodgkin lymphoma (HL) accounts for 8.6-13\% of all lymphoma in mainland China [3,4]. Currently, more than $80 \%$ of patients with newly diagnosed HL are likely to be cured. Patients with early-stage HL commonly receive abbreviated courses of chemotherapy followed by involved-field radiation therapy (IFRT). In contrast, patients with advancedstage HL commonly receive prolonged courses of combination chemotherapy, with radiation therapy used only in selected cases.

For relapsed or refractory (R/R) patients who are ineligible for HDC/ASCT or those in whom HDC/ASCT have failed, treatment with brentuximab vedotin and anti-programmed death 1 (PD-1) antibodies could be considered. 
The phase II studies of pembrolizumab and nivolumab achieved overall response rates (ORR) of 69.0 and $66.3 \%$ in patients with R/R HL, respectively [5, 6]. In Hong Kong China, Chan et al. [7] indicated low-dose pembrolizumab was highly efficacious with minimal toxicity in five patients with R/R classical HL. Pembrolizumab was given with a median dose of $100 \mathrm{mg}$ every 3 weeks and the ORR was $100 \%$ [7]. Nivolumab used in a low dose might similarly be efficacious. A patient received nivolumab at a fixed dose of $40 \mathrm{mg}$ (about $0.55 \mathrm{mg} / \mathrm{kg}$ ) every 2 weeks [8]. Complete remission (CR) was achieved after a cumulative dose of only $2.2 \mathrm{mg} / \mathrm{kg}$ [8]. Several anti-PD-1 antibodies are also developed in mainland China, such as IBI308 (NCT03114683), SHR-1210 (NCT0315425) and BGB-A317 (NCT03209973). Patients with R/R classical HL could participate in clinical trials to receive these anti-PD-1 antibodies treatment. The results of these clinical trials are worthy of expectation.

\section{Non-Hodgkin's lymphoma}

\section{Diffuse large B cell lymphoma}

In China, the most common subtype lymphoma is DLBCL $[3,4]$. The initial treatment for patients with DLBCL is based on the histologic characteristics, the stage, the primary site and other prognostic factors. The most important advance in the management of DLBCL in the past two decades was the addition of rituximab to an anthracycline-containing chemotherapy regimen. The real-world study of rituximab plus chemotherapy as first-line treatment in Chinese patients with DLBCL showed the ORR and CR/unconfirmed CR $(\mathrm{CRu})$ were 94.2 and $73.2 \%$, respectively [9]. According to the retrospective study of Shanghai Lymphoma Research Group, there were significant differences between R-CHOP (rituximab, cyclophosphamide, doxorubicin, vincristine and prednisone) and CHOP (cyclophosphamide, doxorubicin, vincristine and prednisone) chemotherapy for overall survival (OS 84.1 vs $70.2 \%, p=0.018$ ) and progression-free survival (PFS 81.5 vs $66.7 \%, p=0.015$ ), with the median follow-up of 86 months [10]. Hepatitis B virus (HBV) reactivation is a serious complication for patients with lymphoma treated with rituximab-containing chemotherapy. Antiviral prophylaxis can potentially prevent rituximab-associated HBV reactivation in patients with lymphoma and resolved hepatitis B [11]. Huang et al. [12] found among patients seropositive for the hepatitis B surface antigen with DLBCL receiving R-CHOP chemotherapy, the addition of entecavir compared with lamivudine resulted in a lower incidence of HBV-related hepatitis and HBV reactivation.

Due to the prominent efficacy and good tolerability of rituximab in the treatment of lymphoma, several new antiCD20 monoclonal antibodies (mAbs) have been developed.
Ofatumumab and obinutuzumab have been approved for the treatment of chronic lymphocytic leukemia (CLL) [13, 14]. In mainland China, the phase Ib study demonstrated obinutuzumab exposure was comparable in CLL, DLBCL and follicular lymphoma (FL) patients [15]. Pharmacokinetic characteristics of Chinese patients with B cell lymphomas are similar to those in non-Chinese patients [15]. SCT400 is a recombinant, human-mouse chimeric anti-CD20 IgG1 $\mathrm{mAb}$. Phase I study demonstrated that SCT400 was safe and well tolerated in Chinese patients with $\mathrm{CD} 20+\mathrm{B}$ cell nonHodgkin's lymphoma (NHL) [16]. The pharmacokinetics and pharmacodynamics results of SCT400 were comparable to rituximab, and the preliminary efficacy data were encouraging [16]. HLX01 is a rituximab biosimilar produced in mainland China. The multicenter, randomized, phase III studies to compare the efficacy and safety of HLX01 (NCT02787239) or SCT400 (NCT02772822) with rituximab plus $\mathrm{CHOP}$ are ongoing in previously untreated subjects with CD20+ DLBCL.

Up-front HDC/ASCT following R-CHOP could improve the outcome of high-intermediate and high-risk DLBCL patients [17]. Absolute monocyte count is a prognostic indicator in patients with DLBCL after HDC/ASCT [18]. Dose-adjusted CHOP and ICE-based regimens are effective for autologous peripheral blood stem cell (APBSC) mobilization $[19,20]$. Etoposide given at a dose of either 1.0 or $1.5 \mathrm{~g} / \mathrm{m}^{2}$ in combination with recombinant human granulocyte colony-stimulating factor (rhG-CSF) is also an effective and tolerable regimen for APBSC mobilization [21]. CBV (cyclophosphamide, carmustine and etoposide), BEAM (carmustine, etoposide, cytarabine and melphalan) and BEAC (carmustine, etoposide, cytarabine and cyclophosphamide) regimens are all optional high-dose chemotherapy before ASCT for NHL patients [22]. Shi et al. [22] demonstrated the estimated 5-year PFS in the CBV group (43.8\%) was relatively inferior to the BEAM (66.7\%) and BEAC (67.5\%) groups, but the differences were not significant.

Chimeric antigen receptor (CAR)-engineered $\mathrm{T}$ cells are widely studied for cancer immunotherapy. Autologous $\mathrm{T}$ cells with re-engineered chimeric antigen receptors (CAR-T) have been successfully used for some patients with leukemia and lymphoma [23-26]. The multicenter ZUMA-1 phase I study showed autologous CD3 $/$ /CD28-based CAR-T therapy was safe in patients with refractory DLBCL [25]. The ORR was $71 \%(n=5 / 7)$ and CR rate was $57 \%(n=4 / 7)$ [25]. In the ZUMA-1 phase II study, the ORR was 76\% (47\% CR and $29 \%$ PR) at the time of report in the cohort 1 of 51 patients [26]. In recent years, more and more clinical trials from mainland China are registered at ClinicalTrials.gov. According to Liu et al. [27], there are 121 trials of CAR-T cells reported and/or registered at ClinicalTrials.gov for different cancer types from mainland China. The most common type of diseases in CAR-T trials are B cell malignancies [27]. 


\section{Follicular lymphoma}

FL is the most prevalent indolent NHL. The management of FL is mainly determined by histologic grading, clinical stage and tumor burden. For patients with stage I and II, the IFRT is recommended and usually results in longlasting remission. While for patients with stage III and IV, systemic therapy could be taken into consideration. The watchful waiting is still an option for patients without symptoms or/and low tumor burden.

Hou et al. [28] compared the efficacy and safety of RFT (rituximab, fludarabine and pirarubicin) with RCTVP (rituximab, cyclophosphamide, pirarubicin, vindesine and prednisone) in 248 indolent B cell NHL patients in mainland China. There were no statistically significant differences in OS between treatment groups [28]. Compared with RCTVP regimen, RFT regimen was associated with superior PFS both in previously untreated and R/R patients [28]. But grades 3 and 4 hematological adverse events were more common in the RFT group [28]. The efficacy and safety of rituximab and bortezomib were evaluated in 60 Chinese patients with R/R indolent B cell NHL, including FL grades $1-2(n=35)$, CLL $(n=16)$ and marginal zone lymphoma $(n=9)$ [29]. The ORR was $70.0 \%$, with a CR/CRu rate of $31.7 \%$ [29]. The 2-year OS and PFS rates of all patients were 75.0 and $41.0 \%$, respectively [29].

\section{Mantle cell lymphoma}

Mantle cell lymphoma (MCL) may have two different subtypes, which are indolent or aggressive. Asymptomatic indolent MCL patients with low tumor burden can be closely observed, deferring therapy to the time of disease progression. Currently, the recommended first-line treatment for young transplant eligible patients is cytarabine/ rituximab-based combination chemotherapies followed by HDC/ASCT $[30,31]$. The bendamustine and rituximab (BR) regimen is becoming an popular treatment option among the elderly population $[31,32]$.

Novel agents such as bortezomib, lenalidamide and ibrutinib are now available for the treatment of R/R disease. Bruton's tyrosine kinase (BTK) has been defined as a major mediator on B cell-receptor signaling pathway. A phase II study to evaluate the efficacy and safety of BGB-3111, a BTK inhibitor developed in mainland China, in patients with R/R MCL is ongoing (NCT03206970). Another BTK inhibitor CT-1530 is in phase I study for patients with R/R B cell NHL, CLL or Waldenstrom's macroglobulinemia (NCT02981745). Wu et al. [33] discovered a selective and potent BTK/mitogen-activated protein kinase interacting kinase $(\mathrm{MNK})$ dual kinase inhibitor (QL-X-138) through a structure-based drug design approach. QL-X-138 exhibits covalent binding to BTK and non-covalent binding to MNK [33]. Compared to the BTK kinase inhibitor (PCI-32765) and the MNK kinase inhibitor (cercosporamide), QL-X-138 displays a stronger anti-proliferative effect against a variety of B cell cancer cell lines [33]. In Hong Kong China, Gill et al. [34] found oral arsenic trioxide-based regimen was effective with minimal toxicity for R/R MCL. Thirty-nine patients ineligible for HDC/ASCT were treated with arsenic trioxide, chlorambucil and ascorbic acid [34]. The ORR was $49 \%$ with a CR rate of $28 \%$ [34]. The median PFS and OS were 16 and 38 months, respectively [34].

\section{Peripheral T cell lymphomas}

Peripheral T cell lymphoma (PTCL) makes up 23-27\% of all NHL cases in mainland China [3, 4], much higher than that in Western countries of $10-15 \%$ [35]. The most common subtype of PTCL in mainland China is ENKTL, followed by PTCL not otherwise specified (PTCL-NOS), anaplastic large cell lymphoma (ALCL) and angioimmunoblastic T cell lymphoma (AITL) $[3,4]$. PTCL represents a relatively rare group of heterogeneous NHL with a very poor prognosis. The anthracycline-based conventional chemotherapy remains standard treatment, but most patients are either refractory to initial therapy or eventually relapse. A systematic review and meta-analysis of front-line anthracyclinebased chemotherapy for PTCL patients showed the CR rate ranged from $35.9 \%$ for enteropathy-type $\mathrm{T}$ cell lymphoma (ETTL) to $65.8 \%$ for ALCL [36]. The 5-year OS rate was $38.5 \%$ for all PTCL patients [36]. Frontline consolidation treatment with HDC/ASCT was associated with favourable outcomes in patients with PTCL [37-40]. CR to induction chemotherapy was a prognostic factor for survival [37-40].

In mainland China, some new regimens were explored as first-line therapy against PTCL. Li et al. [41] compared the efficacy and safety of GDPT (gemcitabine, cisplatin, prednisone and thalidomide) with standard CHOP regimen for patients with newly diagnosed PTCL. 103 patients were randomly allocated into the GDPT group or CHOP group [41]. Patients receiving GDPT chemotherapy achieved significant higher 2-year PFS rate (57 vs $35 \%, p=0.0035)$ and OS rate $(71$ vs $50 \%, p=0.0001)$ than those receiving CHOP chemotherapy [41]. The CR rate (52 vs $33 \%, p=0.0001)$ and the ORR (67 vs. $49 \%$, $p=0.046)$ in the GDPT group were higher than in the CHOP group [41]. Haemocytopenia was the predominant adverse effect [41]. Endostatin (endostar) is an endogenous inhibitor of angiogenesis. Zhang et al. [42] explored the efficacy and safety of recombinant human endostatin in 
combination with CHOP in 15 PTCL patients. The ORR was $80 \% ; 53.3 \%$ of patients achieved CR [42]. The CR rate was $100 \%$ (3/3) in AITL patients compared to only $36.4 \%$ (4/11) in PTCL-NOS patients [42]. The 5-year PFS and OS rates were 53 and $60 \%$, respectively [42]. Grade 3-4 neutropenia was $86.7 \%$ [42].

The most significant progress in recent years has been the evaluation of novel agents in R/R PTCL, including: agents targeting the signal transduction pathways, agents of immunotherapy, histone deacetylase inhibitors, antifolate and nucleoside analogs. Thus, four agents (pralatrexate, brentuximab vedotin, romidepsin and belinostat) have been approved for the treatment of R/R PTCL by the US Food and Drug Administration (FDA). Chidamide (CS055) is a novel and orally active benzamide class of histone deacetylase inhibitor (HDACi) that selectively inhibits activity of HDAC1, 2, 3 and 10 [43, 44]. The pivotal phase II trial of Chidamide enrolled 83 Chinese patients with $R / R$ PTCL, and 79 patients were eligible for efficacy assessment [45]. The ORR was $28 \%$ including $14 \%$ with CR/CRu [45]. Median PFS and OS were 2.1 and 21.4 months, respectively [45]. AITL patients tended to have higher ORR (50\%) and $\mathrm{CR} / \mathrm{CRu}$ rate (40\%) [45]. Grade 3 to 4 adverse events were mainly thrombocytopenia (22\%), leucopenia (13\%) and neutropenia (11\%), respectively [45]. The multicenter realworld study of Chidamide in $383 \mathrm{R} / \mathrm{R}$ PTCL patients confirmed the favorable efficacy and acceptable safety profile [46]. For patients receiving Chidamide monotherapy and Chidamide combined with chemotherapy, the ORR were 39.06 and 51.18\%, respectively [46]. The China Food and Drug Administration (CFDA) has approved Chidamide for the treatment of R/R PTCL in December 2014. Novel agents approved by FDA or CFDA are summarized in Table 1 [45, 47-50].

In recent years, a variety of interesting novel combinations are also emerging. Several studies have evaluated the addition of a novel agent to CHOP-(like) chemotherapy in the front-line therapy [51-53]. The clinical trials of Chidamide combined with CHOP chemotherapy for newly diagnosed PTCL (NCT02809573) and Chidamide combined with ICE chemotherapy for R/R PTCL (NCT02856997) are ongoing.

\section{Extranodal NK/T cell lymphoma, nasal type}

ENKTL is specific subtype of PTCL with higher prevalence in East Asia. It comprises approximately 50\% of PTCL in mainland China and only accounts for $4.3-5.1 \%$ in north America and Europe $[3,4,35]$. The upper respiratory tract, especially the nasal region, is the most common site of invasion [54]. A multicenter study of 1383 Chinese patients with NKTCL showed $92 \%$ of patients presented with early-stage disease [55]. The 5-year OS rate was $60.3 \%$ for all patients [55]. Stage, age, Eastern Cooperative Oncology Group performance status, lactate dehydrogenase and primary tumor invasion were important prognostic factors [55]. Jiang et al. [56] found Chinese patients with DDX3X mutations presented a poor prognosis. DDX3X mutants exhibited loss of suppressive effects on cell-cycle progression in NK cells, transcriptional activation of NF- $\mathrm{KB}$ and MAPK pathways [56].

Standard treatment for ENKTL patients has not been established. For patients with localized ENKTL, radiotherapy is widely administered and could achieve CR rates of up to $70 \%[57,58]$. However, radiotherapy alone seems inadequate for high-risk patients and the optimal treatment is combined chemoradiotherapy for these patients. In mainland China, some "sandwich" protocols, with earlier RT after an initial 2-3 cycles of chemotherapy followed by further "consolidation" cycles of chemotherapy, achieved good efficacy. The frontline use of "sandwich" chemotherapy regimens included LVP (L-asparaginase, vincristine and prednisone), GELOX (gemcitabine, oxaliplatin and L-asparaginase), P-Gemox (pegaspargase, gemcitabine and oxaliplatin) and LVDP (L-asparaginase, cisplatin, etoposide and dexamethasone) [59-62]. The ORR were 86.4-96.3\%, and the CR rates were 74.1-86.8\% [59-62]. Other studies demonstrated the DICE-L (cisplatin, ifosfamide, etoposide, dexamisone and L-asparaginase) chemotherapy followed by IFRT and

Table 1 Novel agents approved by FDA or CFDA for peripheral T-cell lymphoma

\begin{tabular}{|c|c|c|c|c|c|c|c|}
\hline Agents & Mechanism & Approved diseases & ORR $(\%)$ & $\mathrm{CR}(\%)$ & DOR (months) & OS (months) & References \\
\hline Pralatrexate & Anti-metabolite & R/R PTCL & 29 & 11 & 10.1 & 14.5 & [47] \\
\hline Romidepsin & HDACi & R/R PTCL & 25 & 15 & 17 & NA & [48] \\
\hline Belinostat & HDACi & R/R PTCL & 26 & 11 & 13.6 & 7.9 & [49] \\
\hline Chidamide & HDACi & R/R PTCL & 28 & 14 & 9.9 & 21.4 & {$[45]$} \\
\hline Brentuximab vedotin & Antibody-drug conjugate & $\mathrm{R} / \mathrm{R}$ ALCL & 86 & 57 & 12.6 & NA & {$[50]$} \\
\hline
\end{tabular}

$A L C L$ anaplastic large cell lymphoma, $C R$ complete response, $C F D A$, China Food and Drug Administration, DOR duration of response, FDA U.S. Food and Drug Administration, HDACi histone deacetylase inhibitor, $O S$ overall survival, $O R R$ objective response rate, $P T C L$ peripheral T-cell lymphoma, $R / R$ relapsed or refractory 
Table 2 Treatment for extranodal natural killer/T-cell lymphoma, nasal type in China

\begin{tabular}{|c|c|c|c|c|c|c|}
\hline Disease & Treatment & ORR $(\%)$ & $\mathrm{CR}(\%$ & PFS & OS & Study/references \\
\hline Newly diagnosed stage I/II & Sandwich LVP with RT & 88.5 & 80.8 & 2 years: $80.6 \%$ & 2 years: $88.5 \%$ & Phase II/[59] \\
\hline Newly diagnosed stage I/II & $\begin{array}{l}\text { Sandwich GELOX with } \\
\text { RT }\end{array}$ & 96.3 & 74.1 & 2 years: $86.0 \%$ & 2 years: $86.0 \%$ & Prospective/[60] \\
\hline Newly diagnosed stage I/II & $\begin{array}{l}\text { Sandwich P-Gemox with } \\
\text { RT }\end{array}$ & 92.1 & 86.8 & 1 year: $86.7 \%$ & 1 year: $86.6 \%$ & Retrospective/[61] \\
\hline Newly diagnosed stage I/II & $\begin{array}{l}\text { Sandwich LVDP with } \\
\text { CCRT }\end{array}$ & 86.4 & 83.3 & 3 years: $67.4 \%$ & 3 years: $70.1 \%$ & Phase II/[62] \\
\hline Newly diagnosed stage I/II & DICE-L followed by RT & 100 & 90.9 & 5 years: $82.9 \%$ & $5 y: 89.2 \%$ & Retrospective/[63] \\
\hline Newly diagnosed stage I/II & RT followed by GDP & 95 & 89 & 3 years: $77 \%$ & 3 years: $85 \%$ & Retrospective/[64] \\
\hline $\begin{array}{l}\text { Advanced-stage, relapsed, } \\
\text { or refractory }\end{array}$ & $\begin{array}{l}\text { Modified SMILE vs } \\
\text { CHOP }\end{array}$ & 70 vs. 36 & 45.0 vs. 13 & NA & NA & Retrospective/[66] \\
\hline $\begin{array}{l}\text { Newly diagnosed stage } \\
\text { III/IV }\end{array}$ & DDGP vs SMILE & 95 vs. 67 & 71 vs. 29 & 1 year: $86 \%$ vs. $38 \%$ & 2 years: $74 \%$ vs. $45 \%$ & Prospective/[67] \\
\hline $\begin{array}{l}\text { Advanced-stage, relapsed, } \\
\text { or refractory }\end{array}$ & P-gemox & 80.0 & 51.4 & 3 years: $38.6 \%$ & 3 years: $64.7 \%$ & Retrospective/[68] \\
\hline $\begin{array}{l}\text { Newly diagnosed, } \\
\text { relapsed, or refractory }\end{array}$ & MESA & 87 & 43.5 & $66 \%$ & 2 years: $83.4 \%$ & Phase II/[69] \\
\hline Stage I-IV & DIMG & 74 & 41.7 & NA & NA & Retrospective/[70] \\
\hline
\end{tabular}

$C R$ complete response, $C C R T$ concurrent chemoradiotherapy, $C H O P$ cyclophosphamide doxorubicin vincristine and prednisone, $D D G P$ dexamethasone cisplatin gemcitabine and pegaspargase, DICE- $L$ cisplatin ifosfamide etoposide dexamisone and L-asparaginase, DIMG dexamethasone ifosfamide methotrexate and gemcitabine, GELOX gemcitabine oxaliplatin and L-asparaginase, GDP gemcitabine dexamethasone and cisplatin, $L V D P$ L-asparaginase cisplatin etoposide and dexamethasone, $L V P$ L-asparaginase vincristine and prednisone, MESA methotrexate etoposide dexamethasone and pegaspargase, $R T$ radiotherapy, $N A$ not available, $O R R$ overall response rate, $O S$, overall survival, $P F S$, progression-free survival, P-Gemox pegaspargase gemcitabine and oxaliplatin, SMILE dexamethasone methotrexate ifosfamide L-asparaginase and etoposide

intensity-modulated radiation therapy followed by GDP (gemcitabine, dexamethasone and cisplatin) chemotherapy were both effective for the treatment of newly diagnosed stage I to II ENKTL [63, 64].

The prognoses of patients with stage III and IV disease are extremely poor and the 5-year OS rate is no more than 30\% [55]. Chemotherapy is the main treatment for advanced ENKTL. Conventional CHOP or CHOP-like regimes appeared to be unsatisfactory. Several studies demonstrated that SMILE (dexamethasone, methotrexate, ifosfamide, L-asparaginase and etoposide) regimen is associated with superior efficacy but much higher incidence of grade 4 toxicity [65]. In mainland China, many studies explored effective chemotherapy regimens for advanced-stage or R/R ENKTL. Yang et al. [66] indicated that the modified SMILE chemotherapy regimen markedly improved the OS and PFS rates compared with CHOP chemotherapy regimen. A randomized controlled multicenter study showed DDGP (dexamethasone, cisplatin, gemcitabine and pegaspargase) chemotherapy resulted in significant improvement in PFS, OS, and better tolerability compared with SMILE chemotherapy for newly diagnosed advanced ENKTL patients [67]. Other pegaspargase or gemcitabine-based combination regimens including P-gemox (gemcitabine, oxaliplatin and pegaspargase),
MESA (methotrexate, etoposide, dexamethasone and pegaspargase) and DIMG (dexamethasone, ifosfamide, methotrexate and gemcitabine) also showed high efficacy [68-70]. The studies for the treatment of ENKTL in mainland China are summarized in Table 2. HDC/ASCT in combination with chemoradiotherapy may improve longterm outcomes in patients with newly diagnosed ENKTL [71]. For patients with R/R ENKTL failing L-asparaginase, PD-1 blockade with pembrolizumab is highly effective [72]. IBI308 is also used for the treatment of R/R ENKTL in phase II clinical trial (NCT03228836).

\section{Conclusion}

Although the understanding of the biology of lymphoma has greatly improved, clinical trials are still needed since many unanswered questions remain. In mainland China, HDACi Chidamide, anti-PD-1 antibodies, BTK inhibitors and anti-CD20 antibodies have been developed. Clinical trials are ongoing to use these new agents developed in mainland China for the treatment of HL, DLBCL, MCL and ENKTL (Table 3). These innovative approaches will improve outcomes in this group of diseases. 
Table 3 New agents developed in China for lymphoma treatment

\begin{tabular}{lllll}
\hline Agents & Mechanisms of action & Clinical trial status & Indications & $\begin{array}{l}\text { ClinicalTrials. } \\
\text { gov Identifier }\end{array}$ \\
\hline IBI308 & Anti-PD-1 antibody & Phase II & R/R classical HL & NCT03114683 \\
IBI308 & Anti-PD-1 antibody & Phase II & R/R ENKTL & NCT03228836 \\
BGB-A317 & Anti-PD-1 antibody & Phase II & R/R classical HL & NCT03209973 \\
SHR-1210 & Anti-PD-1 antibody & Phase I/II & R/R HL & NCT03250962 \\
SHR-1210 & Anti-PD-1 antibody & Phase I/II & R/R PMBCL & NCT03346642 \\
SHR-1210 & Anti-PD-1 antibody & Phase II & R/R classical HL & NCT03155425 \\
SCT400 & Anti-CD20 antibody & Phase III & Untreated CD20+ DLBCL & NCT02772822 \\
HLX01 & Rituximab Biosimilar & Phase III & Untreated CD20+ DLBCL & NCT02787239 \\
BGB-3111 & BTK inhibitor & Phase I & R/R B-cell lymphoma & NCT03189524 \\
BGB-3111 & BTK inhibitor & Phase II & R/R non-GCB DLBCL & NCT03145064 \\
BGB-3111 & BTK inhibitor & Phase II & R/R CLL/SLL & NCT03206918 \\
BGB-3111 & BTK inhibitor & Phase II & R/R MCL & NCT03206970 \\
CT-1530 & BTK inhibitor & Phase I & R/R B-NHL, CLL or WM & NCT02981745 \\
\hline
\end{tabular}

$R / R$ relapsed or refractory, $H L$ Hodgkin lymphoma, $P M B C L$ primary mediastinal large B-cell lymphoma, ENKTL extranodal NK/T cell lymphoma, nasal type, DLBCL diffuse large B-cell lymphoma, CLL/SLL chronic lymphocytic leukemia/small lymphocytic lymphoma, $M C L$ mantle cell lymphoma, $W M$ Waldenstrom's macroglobulinemia, $B-N H L$ B cell non-Hodgkin's lymphoma, $P D-1$ programmed death $1, B T K$ bruton's tyrosine kinase

\section{Compliance with ethical standards}

Conflict of interest The author(s) declare that they have no competing interests.

\section{References}

1. Chen W, Zheng R, Zhang S, Zeng H, Xia C, Zuo T, et al. Cancer incidence and mortality in China, 2013. Cancer Lett. 2017;401:63-71.

2. Chen W, Zheng R, Baade PD, Zhang S, Zeng H, Bray F, et al. Cancer Statistics in China, 2015. CA Cancer J Clin. 2016;66(2):115-32.

3. Sun J, Yang Q, Lu Z, He M, Gao L, Zhu M, et al. Distribution of lymphoid neoplasms in China: analysis of 4,638 cases according to the World Health Organization classification. Am J Clin Pathol. 2012;138(3):429-34.

4. Yang QP, Zhang WY, Yu JB, Zhao S, Xu H, Wang WY, et al. Subtype distribution of lymphomas in Southwest China: analysis of 6,382 cases using WHO classification in a single institution. Diagn Pathol. 2011;6:77.

5. Chen R, Zinzani PL, Fanale MA, Armand P, Johnson NA, Brice $\mathrm{P}$, et al. Phase II study of the efficacy and safety of pembrolizumab for relapsed/refractory classic Hodgkin lymphoma. J Clin Oncol. 2017;35(19):2125-32

6. Younes A, Santoro A, Shipp M, Zinzani PL, Timmerman JM, Ansell S, et al. Nivolumab for classical Hodgkin's lymphoma after failure of both autologous stem-cell transplantation and brentuximab vedotin: a multicentre, multicohort, single-arm phase 2 trial. Lancet Oncol. 2016;17(9):1283-94.

7. Chan TS, Luk TH, Lau JS, Khong PL, Kwong YL. Low-dose pembrolizumab for relapsed/refractory Hodgkin lymphoma: high efficacy with minimal toxicity. Ann Hematol. 2017;96(4):647-51.

8. Hwang YY, Khong PL, Kwong YL. Low-dose nivolumab induced remission in refractory classical Hodgkin lymphoma. Ann Hematol. 2017;96(7):1219-20.
9. Wu J, Song Y, Su L, Xu L, Chen T, Zhao Z, et al. Rituximab plus chemotherapy as first-line treatment in Chinese patients with diffuse large B-cell lymphoma in routine practice: a prospective, multicentre, non-interventional study. BMC Cancer. 2016;16:537.

10. Li X, Liu Z, Cao J, Hong X, Wang J, Chen F, et al. Rituximab in combination with CHOP chemotherapy for the treatment of diffuse large B cell lymphoma in China: a 10-year retrospective follow-up analysis of 437 cases from Shanghai Lymphoma Research Group. Ann Hematol. 2012;91(6):837-45.

11. Huang YH, Hsiao LT, Hong YC, Chiou TJ, Yu YB, Gau JP, et al. Randomized controlled trial of entecavir prophylaxis for rituximab-associated hepatitis B virus reactivation in patients with lymphoma and resolved hepatitis B. J Clin Oncol. 2013;31(22):2765-72.

12. Huang H, Li X, Zhu J, Ye S, Zhang H, Wang W, et al. Entecavir vs lamivudine for prevention of hepatitis $\mathrm{B}$ virus reactivation among patients with untreated diffuse large B-cell lymphoma receiving R-CHOP chemotherapy: a randomized clinical trial. JAMA. 2014;312(23):2521-30

13. Goede V, Fischer K, Busch R, Engelke A, Eichhorst B, Wendtner $\mathrm{CM}$, et al. Obinutuzumab plus chlorambucil in patients with CLL and coexisting conditions. N Engl J Med. 2014;370(12):1101-10.

14. Hillmen P, Robak T, Janssens A, Babu KG, Kloczko J, Grosicki S, et al. Chlorambucil plus ofatumumab versus chlorambucil alone in previously untreated patients with chronic lymphocytic leukaemia (COMPLEMENT 1): a randomised, multicentre, open-label phase 3 trial. Lancet. 2015;385(9980):1873-83.

15. Zhai J, Qin Y, Zhu J, Song Y, Shen Z, Du X, et al. Pharmacokinetics of obinutuzumab in Chinese patients with B-cell lymphomas. Br J Clin Pharmacol. 2017;83(7):1446-56.

16. Gui L, Han X, He X, Song Y, Yao J, Yang J, et al. Phase I study of chimeric anti-CD20 monoclonal antibody in Chinese patients with CD20-positive non-Hodgkin's lymphoma. Chin J Cancer Res. 2016;28(2):197-208.

17. Zhao $\mathrm{Y}$, Wang $\mathrm{H}$, Jin $\mathrm{S}$, Zheng J, Huang $\mathrm{M}$, Tang $\mathrm{Y}$, et al. Prognostic analysis of DLBCL patients and the role of upfront ASCT in high-intermediate and high-risk patients. Oncotarget. 2017;8(42):73168-76. 
18. Mo H, Shi Y, Han X, Zhou S, He X, Liu P, et al. Absolute monocyte count is a prognostic indicator in a patient with diffuse large B-cell lymphoma after autologous peripheral blood stem cell transplant. Leuk Lymphoma. 2015;56(2):515-7.

19. Zhou P, Liu P, Zhou SY, He XH, Han XH, Qin Y, et al. Ifosfamide, cisplatin or carboplatin, and etoposide (ICE)-based chemotherapy for mobilization of autologous peripheral blood stem cells in patients with lymphomas. Chin Med J (Engl). 2015;128(18):2498-504.

20. Shi Y, Zhou P, Han X, He X, Zhou S, Liu P, et al. Autologous peripheral blood stem cell mobilization following dose-adjusted cyclophosphamide, doxorubicin, vincristine, and prednisolone chemotherapy alone or in combination with rituximab in treating high-risk non-Hodgkin's lymphoma. Chin J Cancer. 2015;34(11):522-30.

21. Li B, Yang JL, Shi YK, He XH, Han XH, Zhou SY, et al. Etoposide $1.0 \mathrm{~g} / \mathrm{m}^{2}$ or $1.5 \mathrm{~g} / \mathrm{m}^{2}$ combined with granulocyte colonystimulating factor for mobilization of peripheral blood stem cells in patients with malignancy: efficacy and toxicity. Cytotherapy. 2009;11(3):362-71.

22. Shi Y, Liu P, Zhou S, Yang J, Han X, He X, et al. Comparison of $\mathrm{CBV}, \mathrm{BEAM}$ and BEAC high-dose chemotherapy followed by autologous hematopoietic stem cell transplantation in nonHodgkin lymphoma: efficacy and toxicity. Asia Pac J Clin Oncol. 2017;13(5):e423-9.

23. Grupp SA, Kalos M, Barrett D, Aplenc R, Porter DL, Rheingold $\mathrm{SR}$, et al. Chimeric antigen receptor-modified $\mathrm{T}$ cells for acute lymphoid leukemia. N Engl J Med. 2013;368(16):1509-18.

24. Porter DL, Levine BL, Kalos M, Bagg A, June CH. Chimeric antigen receptor- modified $\mathrm{T}$ cells in chronic lymphoid leukemia. N Engl J Med. 2011;365(8):725-33.

25. Locke FL, Neelapu SS, Bartlett NL, Siddiqi T, Chavez JC, Hosing CM, et al. Phase 1 results of ZUMA-1: a multicenter study of KTE-C19 anti- CD19 CAR T cell therapy in refractory aggressive lymphoma. Mol Ther. 2017;25(1):285-95.

26. Neelapu SS, Locke FL, Bartlett NL, Lekakis L, Miklos D, Jacobson CA, et al. Kte-C19 (anti-CD19 CAR T cells) induces complete remissions in patients with refractory diffuse large B-cell lymphoma (DLBCL): results from the pivotal phase 2 Zuma-1. Blood. 2016;128(22):LBA-6.

27. Liu BS, Song YP, Liu DL. Clinical trials of CAR-T cells in China. J Hematol Oncol. 2017;10(1):166.

28. Hou Y, Wang HQ, Ba Y. Comparison on therapeutic effects of RFT and RCTVP regimen in the treatment of patients with indolent B-cell lymphoma in China. Med Oncol. 2012;29(4):2372-8.

29. Yun H, Zhang HL, Wang HQ. Rituximab and bortezomib (RB): a new effective regimen for refractory or relapsed indolent lymphomas. Med Oncol. 2015;32(1):353.

30. Dreyling M, Lenz G, Hoster E, Van Hoof A, Gisselbrecht C, Schmits R, et al. Early consolidation by myeloablative radiochemotherapy followed by autologous stem cell transplantation in first remission significantly prolongs progression-free survival in mantle-cell lymphoma: results of a prospective randomized trial of the European MCL Network. Blood. 2005;105(7):2677-84.

31. Fakhri B, Kahl B. Current and emerging treatment options for mantle cell lymphoma. Ther Adv Hematol. 2017;8(8):223-34.

32. Rummel MJ, Niederle N, Maschmeyer G, Banat GA, von Grünhagen $\mathrm{U}$, Losem C, et al. Bendamustine plus rituximab versus CHOP plus rituximab as first-line treatment for patients with indolent and mantle-cell lymphomas: an open-label, multicentre, randomised, phase 3 non-inferiority trial. Lancet. 2013;381(9873):1203-10.

33. Wu H, Hu C, Wang A. Discovery of a BTK/MNK dual inhibitor for lymphoma and leukemia. Leukemia. 2016;30(1):173-81.

34. Gill H, Au WY, Cheung WW, Lee EY, Kwong YL. Oral arsenic trioxide-based regimen as salvage treatment for relapsed or refractory mantle cell lymphoma. Ann Oncol. 2014;25(7):1391-7.
35. Vose J, Armitage J, Weisenburger D. International peripheral T-cell and natural killer/T-cell lymphoma study: pathology findings and clinical outcomes. J Clin Oncol. 2008;26(25):4124-30.

36. Abouyabis AN, Shenoy PJ, Sinha R, Flowers CR, Lechowicz MJ. A systematic review and meta-analysis of front-line anthracycline-based chemotherapy regimens for peripheral T-cell lymphoma. ISRN Hematol. 2011;2011:623924.

37. Gui L, Shi YK, He XH, Lei YH, Zhang HZ, Han XH, et al. High-dose therapy and autologous stem cell transplantation in peripheral T-cell lymphoma: treatment outcome and prognostic factor analysis. Int J Hematol. 2014;99(1):69-78.

38. Huang H, Jiang Y, Wang Q, Guo L, Jin Z, Fu Z, et al. Outcome of allogeneic and autologous hematopoietic cell transplantation for high-risk peripheral $\mathrm{T}$ cell lymphomas: a retrospective analysis from a Chinese center. Biol Blood Marrow Transplant. 2017;23(8):1393-7.

39. Han X, Zhang W, Zhou D, Ruan J, Duan M, Zhu T, et al. Autologous stem cell transplantation as frontline strategy for peripheral T-cell lymphoma: a single-centre experience. J Int Med Res. 2017;45(1):290-302.

40. Bo J, Zhao Y, Zhang S, Hua W, Wang S, Gao C, et al. Long-term outcomes of peripheral blood stem cell transplantation for 38 patients with peripheral T-cell lymphoma. J Cancer Res Ther. 2016;12(3):1189-97.

41. Li L, Duan W, Zhang L, Li X, Fu X, Wang X, et al. The efficacy and safety of gemcitabine, cisplatin, prednisone, thalidomide versus CHOP in patients with newly diagnosed peripheral T-cell lymphoma with analysis of biomarkers. Br J Haematol. 2017;178(5):772-80.

42. Zhang Q, Cao J, Xue K, Liu X, Ji D, Guo Y, et al. Recombinant human endostatin in combination with CHOP regimen for peripheral $\mathrm{T}$ cell lymphoma. Onco Targets Ther. 2016;10:145-51.

43. Lu X, Ning Z, Li Z, Cao H, Wang X. Development of Chidamide for peripheral T-cell lymphoma, the first orphan drug approved in China. Intractable Rare Dis Res. 2016;5(3):185-91.

44. Ning ZQ, Li ZB, Newman MJ, Shan S, Wang XH, Pan DS, et al. Chidamide (CS055/HBI-8000): a new histone deacetylase inhibitor of the benzamide class with antitumor activity and the ability to enhance immune cell-mediated tumor cell cytotoxicity. Cancer Chemother Pharmacol. 2012;69(4):901-9.

45. Shi Y, Dong M, Hong X, Zhang W, Feng J, Zhu J, et al. Results from a multicenter, open-label, pivotal phase II study of chidamide in relapsed or refractory peripheral T-cell lymphoma. Ann Oncol. 2015;26(8):1766-71.

46. Shi Y, Jia B, Xu W, Li W, Liu T, Liu P, et al. Chidamide in relapsed or refractory peripheral T-cell lymphoma: a multicenter real-world study in China. J Hematol Oncol. 2017;10(1):69.

47. O'Connor OA, Pro B, Pinter-Brown L, Bartlett N, Popplewell L, Coiffier B, et al. Pralatrexate in patients with relapsed or refractory peripheral T-cell lymphoma: results from the pivotal PROPEL study. J Clin Oncol. 2011;29(9):1182-9.

48. Coiffier B, Pro B, Prince HM, Foss F, Sokol L, Greenwood $\mathrm{M}$, et al. Results from a pivotal, open-label, phase II study of romidepsin in relapsed or refractory peripheral T-cell lymphoma after prior systemic therapy. J Clin Oncol. 2012;30(6):631-6.

49. O'Connor OA, Horwitz S, Masszi T, Van Hoof A, Brown P, Doorduijn J, et al. Belinostat in patients with relapsed or refractory peripheral T-cell lymphoma: results of the pivotal phase II BELIEF (CLN-19) study. J Clin Oncol. 2015;33(23):2492-9.

50. Pro B, Advani R, Brice P, Bartlett NL, Rosenblatt JD, Illidge T, et al. Brentuximab vedotin (SGN-35) in patients with relapsed or refractory systemic anaplastic large-cell lymphoma: results of a phase IIstudy. J Clin Oncol. 2012;30(18):2190-6.

51. Fanale MA, Horwitz SM, Forero-Torres A, Bartlett NL, Advani $\mathrm{RH}$, Pro B, et al. Brentuximab vedotin in the front-line treatment 
of patients with CD30+ peripheral T-cell lymphomas: results of a phase I study. J Clin Oncol. 2014;32(28):3137-43.

52. Dupuis J, Morschhauser F, Ghesquieres H, Tilly H, Casasnovas O, Thieblemont $\mathrm{C}$, et al. Combination of romidepsin with cyclophosphamide, doxorubicin, vincristine, and prednisone in previously untreated patients with peripheral T-cell lymphoma: a non-randomised, phase 1b/2 study. Lancet Haematol. 2015;2(4):e160-5.

53. Advani RH, Ansell SM, Lechowicz MJ, Beaven AW, Loberiza F, Carson KR, et al. A phase II study of cyclophosphamide, etoposide, vincristine and prednisone (CEOP) Alternating with Pralatrexate $(\mathrm{P})$ as front line therapy for patients with peripheral T-cell lymphoma (PTCL): final results from the T- cell consortium trial. Br J Haematol. 2016;172(4):535-44.

54. Huang Y, Jia B, Jiang S, Zhou S, Yang J, Liu P, et al. Different clinical characteristics and treatment strategies for patients with localized sinonasal diffuse large B cell lymphoma and extranodal NK/T cell lymphoma. J Hematol Oncol. 2017;10:7.

55. Yang Y, Zhang YJ, Zhu Y, Cao JZ, Yuan ZY, Xu LM, et al. Prognostic nomogram for overall survival in previously untreated patients with extranodal NK/T-cell lymphoma, nasal-type: a multicenter study. Leukemia. 2015;29(7):1571-7.

56. Jiang L, Gu ZH, Yan ZX, Zhao X, Xie YY, Zhang ZG, et al. Exome sequencing identifies somatic mutations of DDX3X in natural killer/T-cell lymphoma. Nat Genet. 2015;47(9):1061-6.

57. Li YX, Fang H, Liu QF, Lu J, Qi SN, Wang H, et al. Clinical features and treatment outcome of nasal-type NK/T-cell lymphoma of Waldeyer ring. Blood. 2008;112(8):3057-64.

58. Li YX, Yao B, Jin J, Wang WH, Liu YP, Song YW, et al. Radiotherapy as primary treatment for stage IE and IIE nasal natural killer/T-cell lymphoma. J Clin Oncol. 2006;24(1):181-9.

59. Jiang M, Zhang H, Jiang Y, Yang Q, Xie L, Liu W, et al. Phase 2 trial of "sandwich" L-asparaginase, vincristine, and prednisone chemotherapy with radiotherapy in newly diagnosed, stage IE to IIE, nasal type, extranodal natural killer/T-cell lymphoma. Cancer. 2012;118(13):3294-01

60. Wang L, Wang ZH, Chen XQ, Li YJ, Wang KF, Xia YF, et al. First-line combination of gemcitabine, oxaliplatin, and L-asparaginase (GELOX) followed by involved-field radiation therapy for patients with stage IE/IIE extranodal natural killer/T-cell lymphoma. Cancer. 2013;119(2):348-55.

61. Jing XM, Zhang ZH, Wu P, Zhang SC, Ren YR, Xiong ZJ, et al. Efficacy and tolerance of pegaspargase, gemcitabine and oxaliplatin with sandwiched radiotherapy in the treatment of newlydiagnosed extranodal nature killer (NK)/T cell lymphoma. Leuk Res. 2016;47:26-31.

62. Jiang M, Zhang L, Xie L, Zhang H, Jiang Y, Liu WP, et al. A phase II prospective study of the "Sandwich" protocol, L-asparaginase, cisplatin, dexamethasone and etoposide chemotherapy combined with concurrent radiation and cisplatin, in newly diagnosed,
I/II stage, nasal type, extranodal natural killer/T-cell lymphoma. Oncotarget. 2017;8(30):50155-63.

63. Dong LH, Zhang LJ, Wang WJ, Lei W, Sun X, Du JW, et al. Sequential DICE combined with 1-asparaginase chemotherapy followed by involved field radiation in newly diagnosed, stage IE to IIE, nasal and extranodal NK/T-cell lymphoma. Leuk Lymphoma. 2016;57(7):1600-6.

64. Huang Y, Yang J, Liu P, Zhou S, Gui L, He X, et al. Intensitymodulated radiation therapy followed by GDP chemotherapy for newly diagnosed stage I/II extranodal natural killer/T cell lymphoma, nasal type. Ann Hematol. 2017;96(9):1477-83.

65. Yamaguchi M, Kwong YL, Kim WS, Maeda Y, Hashimoto C, Suh C, et al. Phase II study of SMILE chemotherapy for newly diagnosed stage IV, relapsed, or refractory extranodal natural killer (NK)/T-cell lymphoma, nasal type: the NK-Cell Tumor Study Group study. J Clin Oncol. 2011;29(33):4410-6.

66. Yang L, Liu H, Xu XH, Wang XF, Huang HM, Shi WY, et al. Retrospective study of modified SMILE chemotherapy for advancedstage, relapsed, or refractory extranodal natural killer $(\mathrm{NK}) / \mathrm{T}$ cell lymphoma, nasal type. Med Oncol. 2013;30(4):720.

67. Li X, Cui Y, Sun Z, Zhang L, Li L, Wang X, et al. DDGP versus SMILE in newly diagnosed advanced natural killer/T-cell lymphoma: a randomized controlled, multicenter, open-label study in China. Clin Cancer Res. 2016;22(21):5223-8.

68. Wang JH, Wang L, Liu CC, Xia ZJ, Huang HQ, Lin TY, et al. Efficacy of combined gemcitabine, oxaliplatin and pegaspargase (P-gemox regimen) in patients with newly diagnosed advancedstage or relapsed/refractory extranodal NK/T-cell lymphoma. Oncotarget. 2016;7(20):29092-101.

69. Liang R, Gao GX, Chen JP, Wang JS, Wang XM, Zeng Y, et al. A phase 2 study of methotrexate, etoposide, dexamethasone, and pegaspargase chemotherapy for newly diagnosed, relapsed, or refractory extranodal natural killer/T-cell lymphoma, nasal type: a multicenter trial in Northwest China. Hematol Oncol. 2016. http s://doi.org/10.1002/hon.2325.

70. Yang JL, Shi YK, Dong M, Dong M, Zhang C, Liu P, et al. A preliminary study of the safety and efficacy of DIMG chemotherapy for natural killer/T-cell lymphoma. Leuk Lymphoma. 2015;56(7):2218-21.

71. Wang J, Wei L, Ye J, Yang L, Li X, Cong J, et al. Autologous hematopoietic stem cell transplantation may improve longterm outcomes in patients with newly diagnosed extranodal natural killer/T-cell lymphoma, nasal type: a retrospective controlled study in a single center. Int J Hematol. 2017. https://doi. org/10.1007/s12185-017-2324-z.

72. Kwong YL, Chan TSY, Tan D, Kim SJ, Poon LM, Mow B, et al. PD1 blockade with pembrolizumab is highly effective in relapsed or refractory NK/T-cell lymphoma failing 1-asparaginase. Blood. 2017;129(17):2437-42. 